\title{
Incidence and Predictors of Loss to Follow-Up Among Pregnant and Lactating Mothers Living with HIV and Enrolled in HIV Care Clinic in Ethiopia: A Systematic Review and Meta-Analysis
}

Asteray Ayenew ( $\square$ amanuelbiruk0077@gmail.com )

Bahir Dar University

Research

Keywords: Loss to Follow-Up, Pregnant and Lactating Mothers, a Systematic Review and Meta-analysis

Posted Date: July 27th, 2021

DOI: https://doi.org/10.21203/rs.3.rs-721419/v1

License: (9) This work is licensed under a Creative Commons Attribution 4.0 International License.

Read Full License 


\section{Abstract}

\section{Background}

Mother-to-Child Transmission of HIV accounts for more than $90 \%$ of all pediatric HIV infections. Ethiopia has recently adopted lifelong antiretroviral therapy (ART) for all HIV-positive pregnant and breastfeeding women (Option B+ strategy), regardless of CD 4 count or clinical stage. Therefore, the aim of this systematic review and meta-analysis was to estimate the pooled incidence and predictors of loss to follow-up among pregnant and lactating mothers living with HIV and enrolled in HIV care clinics in Ethiopia.

Method

The Preferred Reporting Items for Systematic Reviews and Meta-Analyses (PRISMA) guideline was followed. All observational published studies were retrieved using relevant search terms in PubMed, Google Scholar, Cochrane Library, Scopus, African Journals Online, CINHAL, and Ethiopian's university research repository online library. STATA 11 software was used to analyze the data. The Cochrane $Q$ and 12 tests were used to assess the heterogeneity of studies. The pooled estimated prevalence and odds ratios with $95 \%$ confidence intervals were computed by a random-effects model.

Result: The pooled magnitude of loss to follow-up among pregnant and lactating mothers living with HIV and enrolled in HIV care clinics in Ethiopia was 14.28\% (95\% Cl: 10.12-18.44). Poor/fair antiretroviral treatment adherence (Adjusted odds ratio $(A O R)=3.68 ; 95 \%$ Cl: $2.89-4.69)$, who does not know their CD4 level (AOR = 3.68; 95\% Cl: 2.40-5.63), disclosure of HIV status to partner ( $\mathrm{AOR}=2.69 ; 95 \% \mathrm{Cl}$ : 2.08-3.48), experienced drug side effect $(A O R=7.91 ; 95 \% \mathrm{Cl}: 2.94-21.33)$, residing in the rural area $(A O R=2.66 ; 95 \%$ Cl: $2.34-3.03$ ), and no education ( $\mathrm{AOR}=4.28 ; 95 \% \mathrm{Cl}: 3.62-5.00$ ) were the significant predictors of loss to follow up.

\section{Conclusion}

The magnitude of loss to follow-up among pregnant and lactating mothers was high in Ethiopia. Thus, counseling, strengthening the linkage and referral system, establishing appropriate tracing mechanisms, and determining CD4 level is crucial to decrease loss to follow-up among pregnant and lactating mothers living with HIV.

\section{Background}

Worldwide, in 2017, the number of new pediatric HIV infections was reduced from 270,000 in 2009 to 160,000 , and more than $90 \%$ of these children were infected through mother-to-child transmission (MTCT). Without any intervention, the risk of MTCT is $15-30 \%$ during pregnancy and delivery, and 5$20 \%$ during breastfeeding contributing for an overall transmission rate of $20-45 \%$ [1]. The use of combined antiretroviral therapy (ART) and elective caesarean section has reduced MTCT rates to less 
than $2 \%$ in non-breastfeeding populations. Among breastfeeding populations, studies have demonstrated that timely antiretroviral therapy (ART) can reduce Mother To Child Transmission (MTCT) of HIV to $5 \%$ or less [2, 3].

The World Health Organization (WHO) guideline on ART recommends three options to prevent MTCT transmission of HIV infection; Options A, B, and B+. The latest approach, Option B+ PMTCT program, emphasizes the provision of universal, lifelong ART for all HIV-infected women regardless of CD4 count and WHO clinical staging [4]. Moreover, the WHO developed guidelines recommending a 'treat-all' approach, meaning all people diagnosed with HIV should be offered immediate treatment. This has increased the number of women of reproductive age who are receiving ART, regardless of whether they are pregnant or not[4]. However, poor uptake of Prevention of Mother-to-Child Transmission (PMTCT) of HIV services, Loss to Follow-Up (LTFU), and poor adherence to drugs are still a major challenge to achieving virtual elimination of MTCT of HIV especially in Sub-Saharan Africa[4]. Reducing LTFU among mothers initiated on lifelong ART for PMTCT is therefore a crucial step towards the elimination of MTCT of HIV.

The literature shows that high attrition within PMTCT programs could be more of LTFU than of mortality. Cumulative losses in sub-Saharan African PMTCT programs are estimated to range from $20-28 \%$ during antenatal care, up to $70 \%$ at four months postpartum, and close to $81 \%$ at six months after birth $[5,6]$. Many countries are moving towards national coverage of services for PMTCT; however, most children born to women with HIV are not being systematically monitored and followed up during the postpartum period and are thus missing out on life-saving services. The follow-up of known HIV exposed children is not only necessary to identify infants with HIV and to ensure the timely initiation of treatment and care, but to also avoid postpartum HIV transmission and improve overall infant health outcomes. The process of ensuring that all exposed infants and children suspected of being infected with HIV receive an HIV test, and if infected, receive care and treatment, provides an important opportunity for health systems to deliver comprehensive interventions for women and children [7].

One study showed that infants and children starting on antiretroviral therapy (ART) when they were already severely immunodeficient, never regained normal levels of immune functioning even after five years of treatment[8]. Another study showed that such infants and children are more likely to die than those children who received treatment at an earlier stage[9]. Very few children under the age of one are currently being diagnosed and subsequently receiving treatment.

The PMTCT service also ensures the administration of short-course antiretroviral treatment to those identified as HIV positive and their exposed infants [10], provision of continuous posttest counseling and support for exclusive breastfeeding for 6 months, continuous follow-up of mother-child pairs through routine health services including the provision of cotrimoxazole prophylaxis for opportunistic infections for mother and baby [11], and referral to community-based psychosocial support and home-based care services [12]. The United Nations launched the global plan towards the elimination of new HIV infections 
among children by 2015 and keeping their mothers alive. The plan focuses on reaching pregnant women living with HIV and their children from the time of pregnancy until the mothers stop breastfeeding [13].

Follow-up of PMTCT is a key factor in realizing this goal since it ensures retention of PMTCT clients and adherence to PMTCT interventions. The quality and effectiveness of PMTCT services should be assessed on the basis of the number of mother-child pairs who are receiving consistent follow-up and antiretroviral treatment, and the number of confirmed HIV negative children born to HIV positive women [14].

In Ethiopia, the Option B+ program was implemented since 2013 as part of its national policy for preventing new HIV infections among children and to improve maternal survival [15]. Under Option $\mathrm{B}^{+}$, all HIV-infected pregnant women will receive universal ART and will continue the treatment for the rest of their life[16], and have the advantages of simplification of ART protection against MTCT in future pregnancies, continuing the prevention benefits against sexual transmission to serodiscordant partners, avoiding "stop start-stop" approach of antiretroviral drugs, and minimize the opportunity of LTFU [17].

Before the implementation of the Option $\mathrm{B}^{+}$PMTCT program, Ethiopia is one of the top 20 countries, where one child infected with HIV out of three children born to women living with HIV. The proportion of pregnant women living with HIV who received antiretroviral medicines for the prevention of mother-tochild transmission has increased from a baseline of $37 \%$ in 2009 to $77 \%$ in 2014 and increased to $80 \%$ in 2017, an estimated 1.4 million infectious among children age 0-14 years was decreased from 2010 to $2017[18,19]$. This indicates a major success for the Option $B^{+}$strategy, and how it enables pregnant women to get antiretroviral therapy.

However, loss to Follow-Up (LTFU), poor adherence to drugs, and poor uptake of prevention of Mother-toChild Transmission (PMTCT) of HIV services are still a major challenge for the successful implementation of the Option $\mathrm{B}^{+}$program in Ethiopia, where the prevalence of HIV is high particularly among reproductive age populations[20, 21].

Moreover, LTFU with the Option B+ decreases women's access to HIV care and treatment, which leads to the advanced stage of HIV, facilitates the vertical transmission of HIV to the newborn, increases maternal HIV/AIDS-related morbidity and mortality, and facilitates the development of drug resistance[20, 22]. Even though the Option $\mathrm{B}^{+}$program has been implemented for the last 7 years, there are no studies and/or documented reports on the incidence of LTFU, and its predictors among pregnant and lactating women on lifelong ART in Ethiopia. Therefore, the aim of this systematic review and meta-analysis was to estimate the pooled prevalence and predictors of loss to follow-up among pregnant and lactating mothers living with HIV and enrolled in HIV care clinics in Ethiopia and associated factors.

\section{Methods}

This systematic review and meta-analysis were conducted to estimate the pooled prevalence and predictors of lost to follow-up among pregnant and lactating mothers living with HIV and enrolled in HIV 
care clinics in Ethiopia and associated factors. We used the Preferred Reporting Items for Systematic Reviews and Meta-Analyses (PRISMA) checklist guideline[23] (Additional file 1).

Search strategy

First, the PROSPERO database and database of abstracts of reviews of effects (DARE)

(http://www.library.UCSF.edu) were searched to check whether published or ongoing projects exist related to the topic. International Online databases PubMed, EMBASE, Science Direct Cochrane library, HINARI, Google Scholar, CINHAL, SCOPUS, African Journals, and WHO's Global Health Library were used. Different MeSH terms and search engines included "loss to follow-up" OR "PMTCT" OR "loss to follow-up during pregnancy" OR" option B+ discontinuation," OR "PMTCT service dropout," AND "among postpartum women living with HIV/AIDS," OR "among pregnant women living with HIV/AIDS" or "among breastfeeding mothers living with HIV/AIDS" AND related in Ethiopia. Searching terms were based on adapted PICO principles to search through the above-listed databases to access the relevant articles. For unpublished studies, the official website of Ethiopian's University research repository online library (University of Gondar and Addis Ababa University) were used.

Inclusion and exclusion criteria

Inclusion criteria

Studies will be included if they fulfill the following eligibility criteria:

- Study designs: All comparative epidemiological studies (cross-sectional, case-control \& cohort)

- Study settings: studies conducted in Ethiopia

- Participants: pregnant and/or breastfeeding women living with HIV

- Outcome Measures: loss to follow-up HIV care/Option B ${ }^{+}$/ PMTCT services and determinant factors

- Publication Status: All published and unpublished studies

- Data were published: All dates

- Language of Articles: English language only

Exclusion Criteria

- Studies available only as abstract with unclear outcomes, commentaries, editorials, and reviews were excluded. Additionally, qualitative studies and studies conducted in non-English languages will be excluded.

Quality assessment

After collecting the findings from all databases, the articles were exported to a Microsoft Excel spreadsheet. The authors (AAA) independently extracted the data, reviewing the screened and eligible articles. The methodological quality of each study (sampling strategy, response rate, and 
representativeness of the study), comparability, and outcome were checked using the NOS tool. Newcastle-Ottawa Quality Assessment Scale (NOS) for cross-sectional cohort case-control studies was used to assess the methodological quality of a study, and to determine the extent to which a study has addressed the possibility of bias in its design, conduct and analysis. All included articles scored (NOS) 7 and more can be considered as "good" studies with low risk.

Operational definition

- Loss To Follow Up: women who started a lifelong ART and were not seen within 3 months of the last documented visit under Option B+ PMTCT, and not recorded as 'dead' or 'transferred-out' on patient PMTCT logbook or medical cards [24-26].

- Lifelong ART: this is an approach recommended by the World Health Organization to prevent motherto-child HIV transmission in which all HIV-positive pregnant and lactating women are initiated on antiretroviral therapy (ART) for life regardless of CD4 count or the World Health Organization staging.

\section{Data extraction}

Microsoft Excel (2016), and Stata version 11.0 (Stata Corporation, College Station, Texas, USA) software were used for data entry and analysis, respectively. During data extraction; the name of the author, sample size, publication year, study design, prevalence, response rate, population outcome, study site, and different contributing factors were included. Moreover, the incidence of loss to follow-up among pregnant and lactating women with $95 \% \mathrm{Cl}$ and associated factors were collected.

Statistical analysis

To obtain the pooled incidence of loss to follow-up among pregnant and lactating women, a metaanalysis using the random effects DerSimonian and Laird model was performed due to anticipated heterogeneity[27]. Cochran's $Q$ chi-square statistics and $I^{2}$ statistical test was conducted to assess the random variations between primary studies[28]. In this study, heterogeneity was interpreted as $\mathrm{I}^{2}$ value of $0 \%=$ no heterogeneity, $25 \%=$ low, $50 \%=$ moderate, and $75 \%=$ high[29]. In the case of high heterogeneity, subgroup analysis and sensitivity analyses were run to identify possible moderators of this heterogeneity. Potential publication bias was assessed by visually inspecting funnel plots and objectively using the Egger bias test[30]. To account for any publication bias, we used the trim-and-fill method, based on the assumption that the effect sizes of the studies are normally distributed around the center of a funnel plot. The meta-analysis was performed using the Stata version 11.0 (Stata Corporation, College Station, Texas, USA) software. Finally, for all analyses, $P<0.05$ was considered statistically significant.

\section{Result}

\section{Characteristics of the included studies}


Studies of 434 were retrieved from PubMed, Scopus, HINARI, Google Scholar, African Journals, WHO's Global Health Library, and other gray and online repositories accessing articles regarding the prevalence and determinant factors of cervical cancer screening in Ethiopia. After duplicates were expunged, 268 studies remained. Out of the remaining articles, 106 articles were excluded after review of their abstracts and titles. Therefore, 62 full-text articles were assessed and assessed for inclusion criteria, which resulted in the further exclusion of 54 articles primarily due to inaccessibility of full text and outcome of interest were not reported. As a result, 8 studies were met the inclusion criteria to undergo the final systematic review and meta-analysis (Fig. 1).

Study characteristics

Different factors such as poor/fair antiretroviral treatment adherence, knowing baseline CD4 level, disclosure of HIV status to partners, experienced drug side effects, residing in rural areas, and educational level were included in this study. Eight studies with a total of 5,854 study participants were included in this review. All included articles were facility-based study settings. Regarding the study area, four of the studies were conducted in Amhara region, three in Oromia, and one in Tigray region (Table 1).

The Magnitude of loss to follow-up in HIV care

The pooled magnitude of loss to follow-up among pregnant and lactating mothers living with HIV and enrolled in HIV care clinics in Ethiopia is presented with a forest plot (Fig. 2). Therefore, the national estimated magnitude of loss to follow-up in Ethiopia was $14.28 \% \%\left(95 \% \mathrm{Cl}: 10.12-18.44, \mathrm{I}^{2}=91.9 \%, \mathrm{P}<\right.$ $0.001)$.

Publication bias

The funnel plot was assessed for asymmetry distribution of prevalence of lost to follow-up among pregnant and lactating mothers living with HIV and enrolled in HIV care clinics in Ethiopia by visual inspection (Fig. 3). Egger's regression test showed a P value of 0.869 with no evidence of publication bias.

Sensitivity analysis

This systematic review and meta-analysis showed that the point estimate of its omitted analysis lies within the confidence interval of the combined analysis. Therefore, the trim-and-fill analysis was not further computed (Fig. 4).

Subgroup analysis

Subgroup analysis was employed with evidence of heterogeneity. In this study, the Cochrane 12 statistic was $91.9 \%, P<0.001$, which showed evidence of marked heterogeneity. Therefore, subgroup analysis was done using the study region and sample size. As a result, the prevalence of loss to follow-up among 
pregnant and lactating mothers living with HIV and enrolled in HIV care clinics was highest in Amhara $14.8 \%$, whereas $14.48 \%$ in the study conducted with a sample size $>334$ (Figs. 5 and 6 ).

Determinants of lost to follow-up in Ethiopia

Relationship between women's educational level and lost to follow-up

The risk of LTFU among women who had no education was 4.28 times $(\mathrm{OR}=4.28,95 \% \mathrm{Cl}: 3.62-5.00)$ higher than women who had secondary and above education level. In this meta-analysis, the included studies were characterized by the existence of no heterogeneity $\left(\mathrm{I}^{2}=0.0 \%, \mathrm{P}=0.826\right)$ (Fig. 7).

Relationship between women's residency and lost to follow-up

Likewise, the loss to follow-up among women who were residing in rural areas was 2.66 times higher than women who were residing in urban areas $(O R=2.66,95 \% \mathrm{C}: 2.34-3.03)$. The included studies were characterized by the existence of no heterogeneity $\left(I^{2}=0.0 \%, P=0.503\right)$ (Fig. 8).

Relationship between experienced drug side effects and lost to follow-up

The loss to follow-up among women who experienced antiretroviral drug side effects was 7.91 times higher than women who don't experience antiretroviral drug side effect s (OR = 7.91, 95\% Cl: 2.94-21.33). The included studies were characterized by the existence of high heterogeneity $\left(I^{2}=96.4 \%, P<0.001\right)$ (Fig. 9).

Relationship between disclosure of HIV status to partners and lost to follow-up

The risk of loss to follow-up among women who did not disclose their status to their sexual partners was 2.69 times higher than women's disclose their status ( $\mathrm{OR}=2.69,95 \% \mathrm{Cl}: 2.08-3.48)$. In this Metaanalysis and systematic review, the included studies were characterized by the existence of high heterogeneity $\left(I^{2}=94.8 \%, P<0.001\right)$ (Fig. 10).

Relationship between poor treatment adherences and lost to follow-up

The risk of loss to follow-up among pregnant and lactating women who were on ART and had Poor/fair antiretroviral treatment adherence was 2.69 times higher than women who had good adherence $(\mathrm{OR}=$ 3.68; 95\% Cl: 2.89-4.69). In this Meta-analysis and systematic review, the included studies were characterized by the existence of moderate heterogeneity $\left(I^{2}=77.4 \%, P=0.012\right)$ (Fig. 11).

Relationship between knowing baseline CD4 level and lost to follow-up

The risk of loss to follow-up among women who do not know their baseline CD4 level was 3.68 times higher than women who know their status $(\mathrm{OR}=3.68 ; 95 \% \mathrm{Cl}$ : 2.40-5.63). In this Meta-analysis and systematic review, the included studies were characterized by the existence of high heterogeneity $\left(1^{2}=\right.$ $97.5 \%, P<0.001$ ) (Fig. 12). 


\section{Discussion}

The risk of MTCT is $15-30 \%$ during pregnancy and delivery, and $5-20 \%$ during breastfeeding, contributing to an overall transmission rate of $20-45 \%[38]$. The World Health Organization (WHO) guideline on ART recommends three options to prevent MTCT transmission of HIV infection-Options A, $\mathrm{B}$, and $\mathrm{B}+$. The latest approach, Option B+ PMTCT program, emphasizes on the provision of universal, lifelong ART for all HIV-infected women regardless of CD4 count and the WHO clinical staging [39]. Moreover, WHO developed guidelines recommending a 'treat-all' approach, meaning all people diagnosed with HIV should be offered immediate treatment. This has increased the number of women of pregnant and lactating mothers for their life, for their infants in the womb and newborns[40].

In this review, eight studies comprising a total of 5, 854 participants were analyzed to estimate the best available evidence for the prevalence and factors associated with loss to follow-up among pregnant and lactating mothers living with HIV and enrolled in the HIV care clinics in Ethiopia. Accordingly, the pooled prevalence of loss to follow-up among pregnant and enrolled in HIV care clinics in Ethiopia was $14.28 \% \%$ (95\% Cl: 10.12-18.44). The result is lower than the study findings in rural Uganda 37\% [41], Kenya [42] $31.5 \%$, and Malawi [43] 19\%. The possible reason might be Ethiopia has been implementing the Option $\mathrm{B}^{+}$ approach as a part of its national policy for preventing new HIV infection of newborns and to improve maternal health. Moreover, all pregnant and lactating women receive universal ART thought their life which avoids a stop-start-stop approach of antiretroviral drugs and minimizes the opportunity of loss to follow-up[42]. However, the result is higher than studies conducted in Papua, New Guinea[44] 10.2\%, and England[45] 12.5\%. The possible reason for this variation could be due to differences in the sociodemographic and economic status of the study participants as well as the countries' health policy variations like the institutional framework to promote ART adherence, which could have largely succeeded in implementing successful programs to loss to follow-up.

The study also identified different predictors of loss to follow-up from the program among pregnant and lactating mothers. This research revealed that women's level of education is an implication in loss to follow-up of HIV care. The risk of loss to follow-up among pregnant and lactating mothers with no education was higher than women who had attended secondary and above education. The result is consistent with previous studies of systematic reviews in low and middle-income countries (LMICs)[46], and Uganda [42]. The possible reason might be each additional year in school increases the likelihood of retaining the program. Apparently, higher education levels contribute to better health literacy, self-care and greater access to information about the program.

Women experienced antiretroviral drug side effects were another determinant factor for loss to followup. Losses to follow-up among women who experienced antiretroviral drug side effects were higher than a woman who does't experience antiretroviral drug side effects. The result is in line with studies in Malawi[47], and Nigeria[48]. 
Moreover, consistent with findings in Tanzania[25], Uganda [46], and Malawi[46], the risk of LTFU among women residing in rural was higher as compared to women residing in urban. This might be explained as the distance from home to hospital, particularly in rural areas where women cannot easily get transport services due to physical barriers, forces women to walk long distances, may force them to miss appointments leading to poor adherence to ART.

In the current study, the loss to follow-up among pregnant and lactating women who were on ART and had poor/fair antiretroviral treatment adherence was higher than women who had good adherence. This finding was consistent with the findings of studies conducted in Malawi [49], among pregnant, poor adherence affects the level of follow-up due to fail to follow as per schedule.

The risk of loss to follow-up among women who do not know their baseline CD4 level was higher than women who know their CD4 status. This was consistent with studies conducted in South Africa[50] and Tanzania[51]. This might be related to the feeling of healthiness, fear of side effects of ART treatment, afraid to disclose their HIV status, stigma, and discrimination related to the disease.

HIV disclosure status is the other predictor of loss to follow-up. The risk of loss to follow-up among women who did not disclose their status to their sexual partners was higher than women who disclose their status. The result is supported by studies done in Uganda [52], Indonesia [53], Sub-Saharan Africa [54], and Tanzania[46]. This might be related to the stigma associated with the disease, fear of negative consequences from their partners, and perceiving to preserve family stability.

\section{Conclusion}

The magnitude of loss to follow-up among pregnant and lactating mothers was high in Ethiopia. Rural residency, being uneducated, not disclosing their HIV status, not knowing the baseline CD 4 count, experienced ART drug side effects, and poor/fair level of ART adherence were significantly associated with LTFU.

Thus, to decrease LTFU, it is better to integrate preventive health education not only for pregnant women and their partners but also for the general public. This will help to raise awareness and openness in discussing HIV infection and PMTCT interventions and to lessen the stigma and fear surrounding HIV infection. Provision of continuous training for PMTCT providers and community workers is also needed to ensure quality services in PMTCT programs including CD4 count determining and managing drug side effects. Lack of HIV results from disclosure to male partners by HIV-positive pregnant and lactating mothers compounded by low male partner involvement in PMTCT programs is bringing about an increase in loss to follow-up in PMTCT programs and strategies such as extending clinic hours to accommodate men and incorporating them in PMTCT is crucial.

\section{Abbreviations}

AIDS: Acquired Immuno-deficiency syndrome 
ART: Antiretroviral therapy

HIV: Human immunodeficiency virus

LTFU: Loss to follow-up

MTCT: Mother-to-Child Transmission

PMTCT: Prevention of Mother-to-Child Transmission

\section{Declarations}

Ethics approval and consent to participate

Not applicable

Consent for publication

Not applicable

Availability of data and materials

The datasets used and/or analyzed during the current study are available from the corresponding author on reasonable request.

Authors' contributions

This research was conducted, analyzed, and approved by Asteray Assmie Ayenew

Competing interests

The author declared that I have no financial and nonfinancial competing interests

Funding

No funding was obtained for this study

Acknowledgment

Not Applicable

\section{References}

1. Chaka, T.E., T.W. Abebe, and R.T. Kassa, Option B+ prevention of mother-to-child transmission of HIV/AIDS service intervention outcomes in selected health facilities, Adama town, Ethiopia. HIV/AIDS (Auckland, N.Z.), 2019. 11: p. 77-82. 
2. C. S. Chasela, M.G.H., D. J. Jamieson, et al., Maternal or infant antiretroviral drugs to reduce HIV-1 transmission. The New England Journal of Medicine, 2010. 362: p. 2271-2281.

3. R. L. Shapiro, M.D.H., A. Ogwu et al., Antiretroviral regimens in pregnancy and breastfeeding in Botswana. The New England Journal of Medicine, 2010. 362: p. 2282-2294.

4. (WHO), W.H.O., Programmatic Update: Use of Antiretroviral Drugs for Treating Pregnant Women and Preventing HIV Infection in Infants. Geneva: WHO. 2012.

5. Painter, T., et al., Sociodemographic factors associated with participation by HIV-1-positive pregnant women in an intervention to prevent mother-to-child transmission of HIV in Cote d'Ivoire. International journal of STD \& AIDS, 2005. 16(3): p. 237-242.

6. Sherman, G., et al., PMTCT from research to reality-results from a routine service. South African Medical Journal, 2004. 94(4): p. 289-292.

7. UNICEF, Scaling up early infant diagnosis and linkages to care and treatment. 2013.

8. Patel, K., et al., Long-term effects of highly active antiretroviral therapy on CD4+ cell evolution among children and adolescents infected with HIV: 5 years and counting. Clinical Infectious Diseases, 2008. 46(11): p. 1751-1760.

9. Arrivé, E., et al. Response to ART in children in sub-Saharan Africa: a pooled analysis of clinical databases, the KIDS-ARTLINC Collaboration [Abstract 727.]. in 14th Conference on Retroviruses and Opportunistic Infections. 2007.

10. Guay, L.A., et al., Intrapartum and neonatal single-dose nevirapine compared with zidovudine for prevention of mother-to-child transmission of HIV-1 in Kampala, Uganda: HIVNET 012 randomised trial. The Lancet, 1999. 354(9181): p. 795-802.

11. Badri, M., et al., Initiating co-trimoxazole prophylaxis in HIV-infected patients in Africa: an evaluation of the provisional WHO/UNAIDS recommendations. AIDs, 2001. 15(9): p. 1143-1148.

12. Perez, F., et al., Implementing a rural programme of prevention of mother-to-child transmission of HIV in Zimbabwe: first 18 months of experience. Tropical medicine \& international health, 2004. 9(7): p. 774-783.

13. UNAIDS, P., World leaders launch plan to eliminate new HIV infections among children by 2015. Press release, 2011.9.

14. Adedimeji, A., et al., A qualitative study of barriers to effectiveness of interventions to prevent motherto-child transmission of HIV in Arba Minch, Ethiopia. International Journal of Population Research, 2012. 2012.

15. UNAIDS, U., Countdown to ZERO: global plan towards the elimination of new HIV infections among children by 2015 and keeping their mother alive. UNAIDS, 2015.

16. O'Brien, L., Shaffer, N., Sangrujee, N. and Abimbola, T.O. , The incremental cost of switching from Option B to Option B+ for the prevention of mother-to-child transmission of HIV. . Bulletin of the World Health Organization, 2014: p. 162-170. 
17. (WHO)., W.H.O., Programmatic Update: Use of Antiretroviral Drugs for Treating Pregnant Women and Preventing HIV Infection in Infants. 2012.

18. UNAIDS, U., and WHO. , The incredible journey of the global plan towards the elimination of new HIV infections among children by 2015 and keeping their mothers alive. Geneva: World Health Organization. 2016.

19. (UNAIDS), U.N.J.H.A.P.P.C.g.b.b.r.i.G.G.A.U., 2018.

20. unicef., Addressing the Global HIV Epidemic among pregnant women, mothers, children and adolescents UNICEF's Global HIV response 2018-2021. Geneva: UNICEF. 2018.

21. Tolossa, T., et al., Incidence and predictors of lost to follow-up among women under Option B+ PMTCT program in western Ethiopia: a retrospective follow-up study. BMC Research Notes, 2020. 13(1): p. 18.

22. Kyaw K, O.M., Kyaw N. , Low mother-to-child HIV transmission rate but high loss-to-follow-up among mothers and babies in Mandalay, Myanmar; a cohort study. . PLoS ON, 2017. 12: p. e0184426.

23. Moher, D., et al., Preferred reporting items for systematic reviews and meta-analyses: the PRISMA statement. PLoS Med, 2009. 6(7): p. e1000097.

24. Bigirimana, F., M.N. Owiredu, and I. Nuwagira, Prevention of mother-to-child transmission technical update: implementing the 'treat all'approach among pregnant and breastfeeding women living with HIV in the who African region. Victoria: Bigirimana, 2016.

25. Kweyamba, M., et al., Loss to follow-up among HIV positive pregnant and lactating mothers on lifelong antiretroviral therapy for PMTCT in rural Uganda. Advances in Public Health, 2018. 2018.

26. Mitiku, I., et al., Factors associated with loss to follow-up among women in Option B+ PMTCT programme in northeast Ethiopia: a retrospective cohort study. Journal of the International AIDS Society, 2016. 19(1): p. 20662.

27. DerSimonian, R. and N. Laird Meta-analysis of clinical trials. Control Clin Trials, 1986. 7(3): p. 177-88.

28. Huedo-Medina, T.B., et al., Assessing heterogeneity in meta-analysis: Q statistic or I2 index? Psychol Methods, 2006. 11(2): p. 193-206.

29. Higgins JP, A.D., Assessing risk of bias in included studies. Cochrane handbook for systematic reviews of interventions:. Cochrane book series 2008: p. 187-241.

30. Egger M, D.-S.G., Altman D. , Systematic reviews in health care: meta-analysis in context John Wiley \& Sons, 2008.

31. Mitiku, I., et al., Factors associated with loss to follow-up among women in Option B+ PMTCT programme in northeast Ethiopia: a retrospective cohort study. J Int AIDS Soc, 2016. 19(1): p. 20662.

32. Yeshialem Asefa E, E.D.D., Level of Good Adherence on Option B+ PMTCT and Associated Factors Among HIV Positive Pregnant and Lactating Mothers in Public Health Facilities of llu Abba Bor and Buno Bedele Zones, Oromia Regional State, Southwestern Ethiopia. HIV AIDS (Auckl). , 2020. 12: p. 789-798. 
33. Demissie, D., M. Mossisa, and M. Haile, Assessment of Loss to Follow-Up (LTFU) and Associated Factors among Pregnant Women Initiated Antiretroviral Under Option B+ in Selected Health Facilities of West Zone Oromia, Ethiopia. 2019. 8: p. 314-321.

34. Mamuye, M., A. Baraki, and M. Kindie, Incidence and Predictors of Loss-to-follow-up Among Pregnant and Breastfeeding Women on Option B+ PMTCT Program in Northwest Ethiopia: a Retrospective Follow-up Study. 2020.

35. Ebuy, H., H. Yebyo, and M. Alemayehu, Level of adherence and predictors of adherence to the Option B+ PMTCT programme in Tigray, northern Ethiopia. Int J Infect Dis, 2015. 33: p. 123-9.

36. Wondimu F, Y.F., Admassu E, Binu W, Bulto GA, Lake G, Girmaye E, Temesgen K, Marama T. , Adherence to Option B+ Care for the Prevention of Mother-to-Child Transmission Among Pregnant Women in Ethiopia. https://doi.org/10.2147/HIV.S282463 HIV AIDS (Auckl), 2020. 12: p. 769-778.

37. MT., L., Adherence to Option B+ and Associated Factors Among Pregnant Women on PMTCT Services at Public Health Facilities of East Shawa Zone, Oromia, Ethiopia. . J Women's Health Reprod Med. , 2018. 1(2).

38. Okoli, J.C. and G.E. Lansdown Barriers to successful implementation of prevention-of-mother-to-child transmission (PMTCT) of HIV programmes in Malawi and Nigeria: a critical literature review study. The Pan African Medical Journal, 2014. 19.

39. Organization, W.H., Programmatic update. Use of Antiretroviral Drugs for Treating Pregnant Women And Preventing HIV Infection in Infants. In. Geneva, 2012.

40. UNAIDS, U., Countdown to ZERO: global plan towards the elimination of new HIV infections among children by 2015 and keeping their mother alive. 2011: UNAIDS.

41. Kweyamba, M., et al., Loss to Follow-Up among HIV Positive Pregnant and Lactating Mothers on Lifelong Antiretroviral Therapy for PMTCT in Rural Uganda. Advances in Public Health, 2018. 2018: p. 7540587.

42. Moth, I.A., A.B.C.O. Ayayo, and D. Kaseje, Assessment of utilisation of PMTCT services at Nyanza Provincial Hospital, Kenya. SAHARA-J: Journal of Social Aspects of HIV/AIDS, 2005. 2(2): p. 244250.

43. Manzi, M., et al., High acceptability of voluntary counselling and HIV-testing but unacceptable loss to follow up in a prevention of mother-to-child HIV transmission programme in rural Malawi: scaling-up requires a different way of acting. Tropical Medicine \& International Health, 2005. 10(12): p. 12421250.

44. Kelly-Hanku, A., et al., Loss to follow up of pregnant women with HIV and infant HIV outcomes in the prevention of maternal to child transmission of HIV programme in two high-burden provinces in Papua New Guinea: a retrospective clinical audit. BMJ Open, 2020. 10(12): p. e038311.

45. Tariq, S., et al., Loss to Follow-Up After Pregnancy Among Sub-Saharan Africa-Born Women Living With Human Immunodeficiency Virus in England, Wales and Northern Ireland: Results From a Large National Cohort. Sexually Transmitted Diseases, 2016. 43(5): p. 283-289. 
46. Hodgson, I., et al., A systematic review of individual and contextual factors affecting ART initiation, adherence, and retention for HIV-infected pregnant and postpartum women. PLoS One, 2014. 9(11): p. e111421.

47. Mpinganjira, S., et al., Factors associated with loss-to-follow-up of HIV-positive mothers and their infants enrolled in HIV care clinic: A qualitative study. BMC public health, 2020. 20(1): p. 298-298.

48. Onoka, C.A., et al., Retention and loss to follow-up in antiretroviral treatment programmes in southeast Nigeria. Pathog Glob Health, 2012. 106(1): p. 46-54.

49. Tweya, H., et al., Loss to follow-up before and after initiation of antiretroviral therapy in HIV facilities in Lilongwe, Malawi. PloS one, 2018. 13(1): p. e0188488.

50. Erlwanger, A.S., et al., Patterns of HIV care clinic attendance and adherence to antiretroviral therapy among pregnant and breastfeeding women living with HIV in the context of Option B+ in Zimbabwe. JAIDS Journal of Acquired Immune Deficiency Syndromes, 2017. 75: p. S198-S206.

51. Phillips, T., et al., Disengagement of HIV-positive pregnant and postpartum women from antiretroviral therapy services: a cohort study. Journal of the International AIDS Society, 2014. 17(1): p. 19242.

52. Kiwanuka, G., et al., Retention of HIV infected pregnant and breastfeeding women on option B+ in Gomba District, Uganda: a retrospective cohort study. BMC infectious diseases, 2018. 18(1): p. 533.

53. Lumbantoruan, C., et al., Understanding women's uptake and adherence in Option B+ for prevention of mother-to-child HIV transmission in Papua, Indonesia: A qualitative study. PloS one, 2018. 13(6): p. e0198329.

54. Gourlay, A., et al., Barriers and facilitating factors to the uptake of antiretroviral drugs for prevention of mother-to-child transmission of HIV in sub-Saharan Africa: a systematic review. J Int AIDS Soc, 2013. 16(1): p. 18588.

\section{Tables}

Table 1; Descriptive summary of studies included in this systematic review and meta-analysis. 


\begin{tabular}{|c|c|c|c|c|c|}
\hline $\begin{array}{l}\text { Author(year of } \\
\text { study) }\end{array}$ & study design(setting) & $\begin{array}{l}\text { Sample } \\
\text { Size }\end{array}$ & $\begin{array}{l}\text { Study } \\
\text { region }\end{array}$ & $\begin{array}{l}\text { Prevalence } \\
(\%)\end{array}$ & $\begin{array}{l}\text { NOS } \\
\text { quality } \\
\text { score }\end{array}$ \\
\hline $\begin{array}{l}\text { Tolossa T.et } \\
\text { al(2020)[21] }\end{array}$ & $\begin{array}{l}\text { a retrospective follow-up } \\
\text { study }\end{array}$ & 393 & Oromia & 15.4 & 9 \\
\hline $\begin{array}{l}\text { Israel M. et al (2016) } \\
\text { [31] }\end{array}$ & $\begin{array}{l}\text { a retrospective cohort } \\
\text { study }\end{array}$ & 418 & Amhara & 16.5 & 9 \\
\hline $\begin{array}{l}\text { Yeshalem A.et } \\
\text { al(2020)[32] }\end{array}$ & $\begin{array}{l}\text { Institutional-based cross- } \\
\text { sectional }\end{array}$ & 190 & Amhara & 18.9 & 8 \\
\hline $\begin{array}{l}\text { Dereje B.et al(2018) } \\
\text { [33] }\end{array}$ & prospective cohort study & 334 & Oromia & 18.2 & 9 \\
\hline $\begin{array}{l}\text { Melkamu M.et al } \\
(2020)[34]\end{array}$ & $\begin{array}{l}\text { retrospective follow- } \\
\text { up study }\end{array}$ & 416 & Amhara & 6.8 & 8 \\
\hline $\begin{array}{l}\text { Tolosa E.et al(2019) } \\
\text { [1] }\end{array}$ & $\begin{array}{l}\text { retrospective follow- } \\
\text { up study }\end{array}$ & 248 & Oromo & 4.2 & 8 \\
\hline $\begin{array}{l}\text { Haftom E.et } \\
\text { al(2015) [35] }\end{array}$ & Cross- sectional study & 277 & Tigray & 13 & 9 \\
\hline $\begin{array}{l}\text { Wondimu F.et al } \\
(2020)[36]\end{array}$ & Cross-sectional study & 347 & Amhara & 19.8 & 9 \\
\hline $\begin{array}{l}\text { Mihratu T.et } \\
\text { al(2018)[37] }\end{array}$ & Cross - sectional study & 304 & Oromo & 17.4 & 9 \\
\hline
\end{tabular}

\section{Supplementary}

Additional File 1 is not available with this version

\section{Figures}




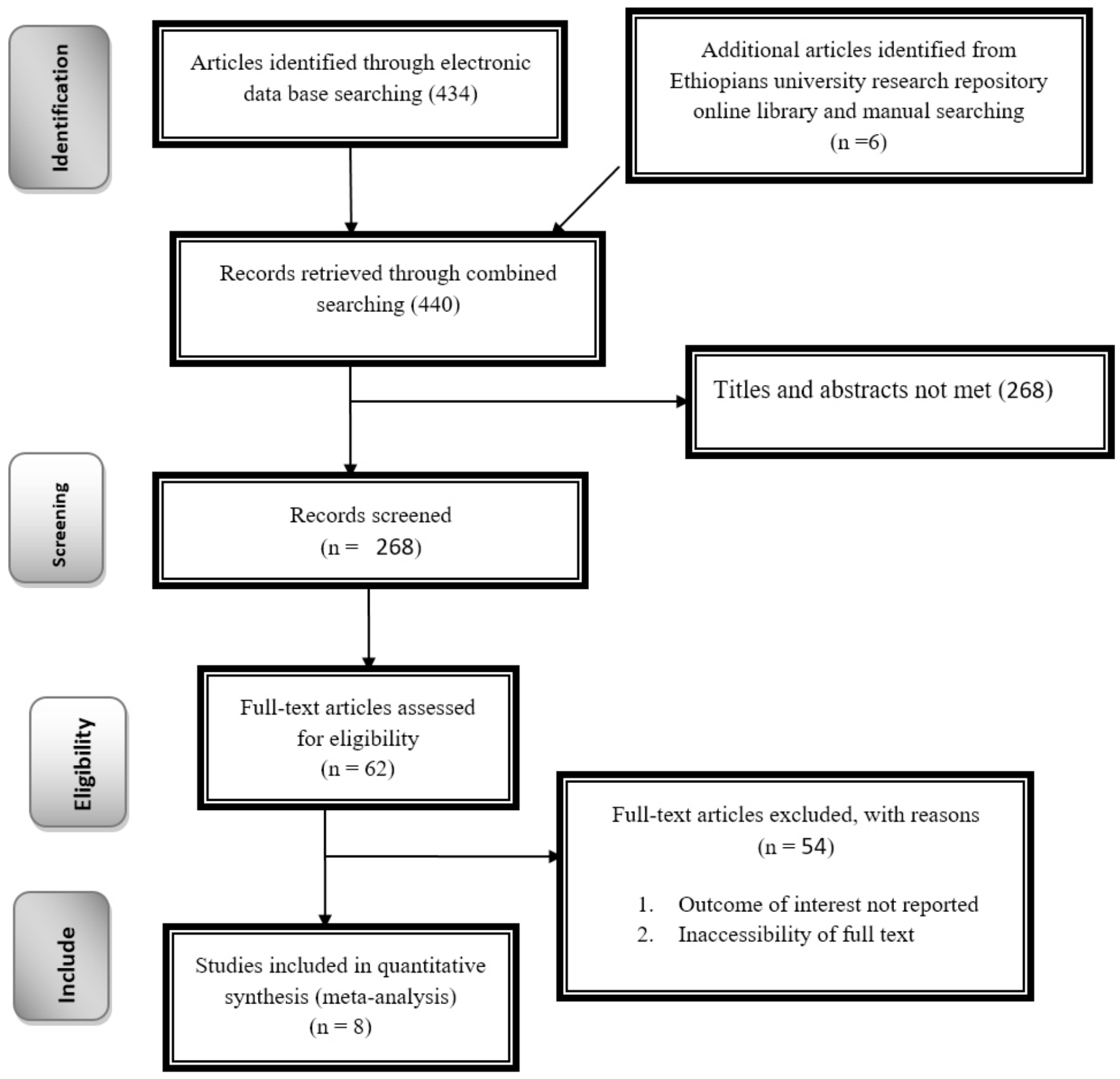

Figure 1

PRISMA Flow diagram for identification and selection of articles for inclusion in the review. 


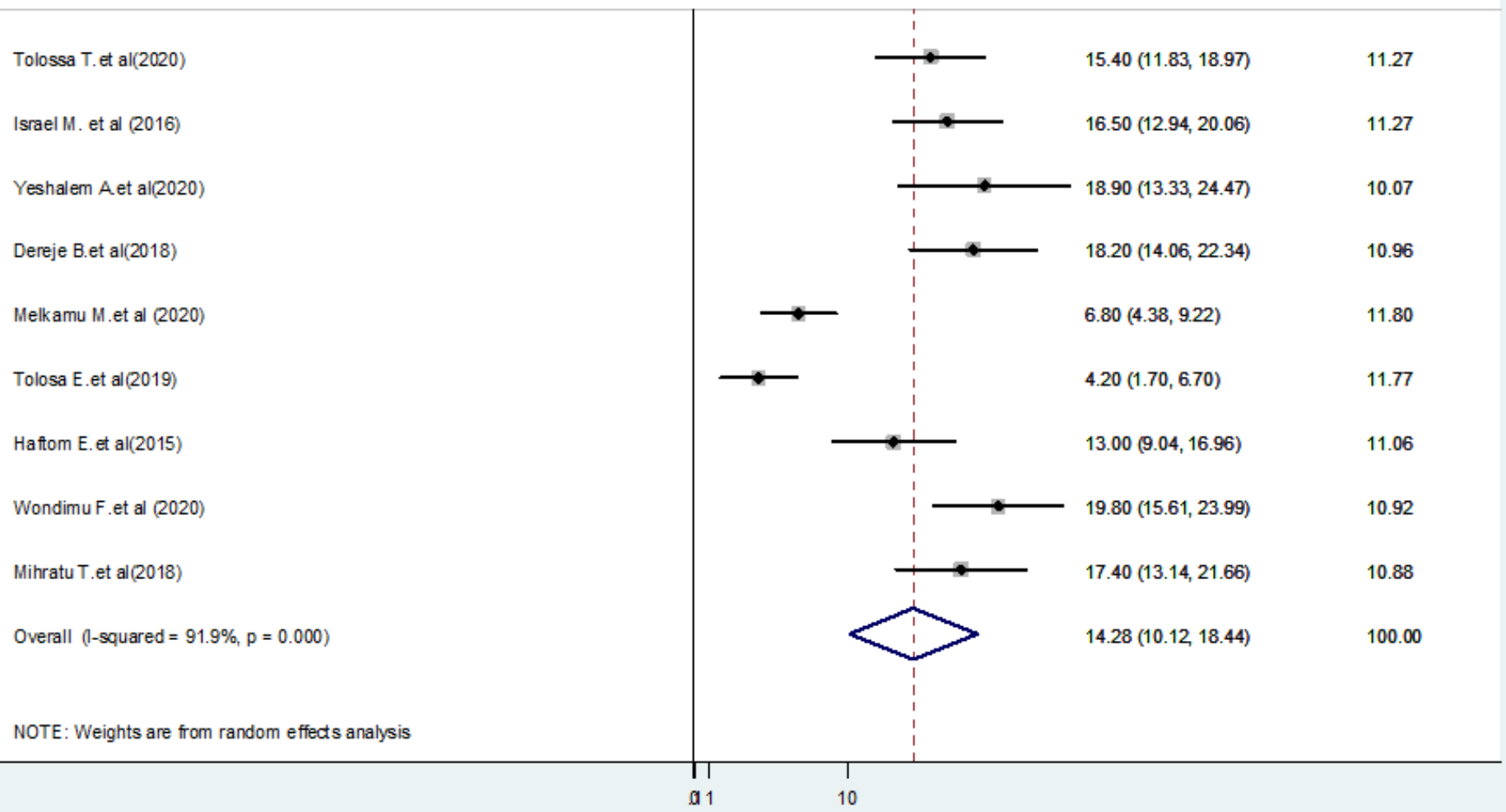

\section{Figure 2}

Forest plot for prevalence of lost to follow-up among pregnant and lactating mothers living with HIV and enrolled in HIV care clinics in Ethiopia, 2020 


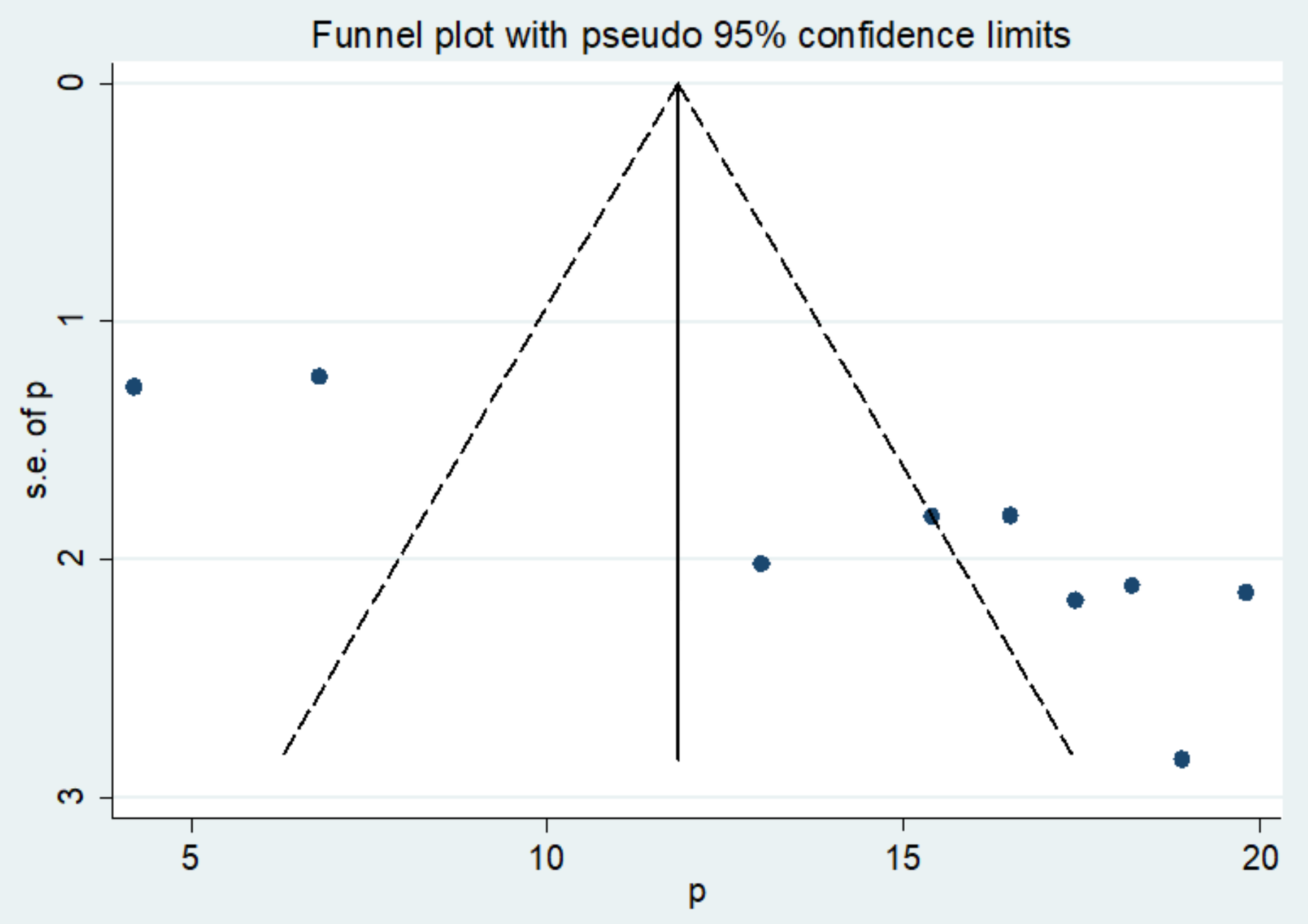

Figure 3

Funnel plot with $95 \%$ confidence limits of the pooled prevalence of lost to follow-up among pregnant and lactating mothers living with HIV and enrolled in HIV care clinics in Ethiopia, 2020 
Meta-analysis estimates, given named study is omitted

Tolossa T.et al(2020)

Israel M. et al (2016)

Yeshalem A.et al(2020)

Dereje B.et al(2018)

Melkamu M.et al (2020)

Tolosa E.et al(2019)

Haftom E.et al(2015)

Wondimu F.et al (2020)

Mihratu T.et al(2018)

| Lower Cl Limit o I Upper Cl Limit

| Lower Cl Limit oEstimate I Upper Cl Limit

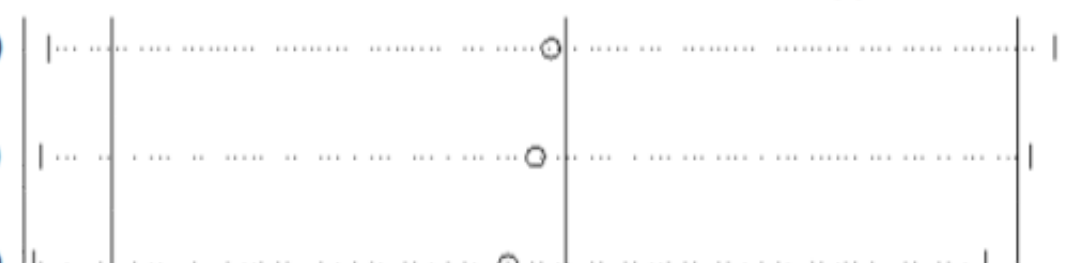

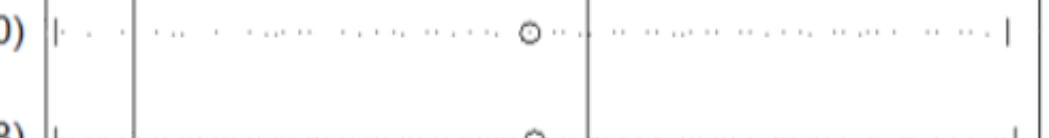

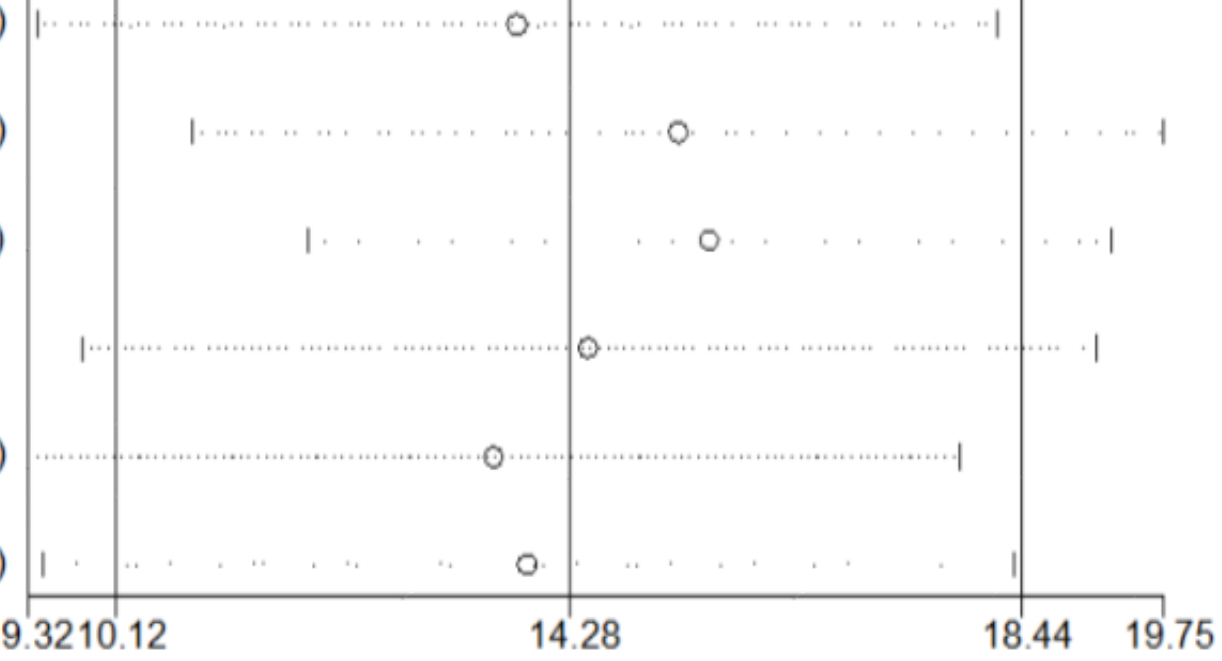

\section{Figure 4}

Sensitivity analysis of the pooled prevalence of lost to follow-up among pregnant and lactating mothers living with HIV and enrolled in HIV care clinics in Ethiopia, 2020 


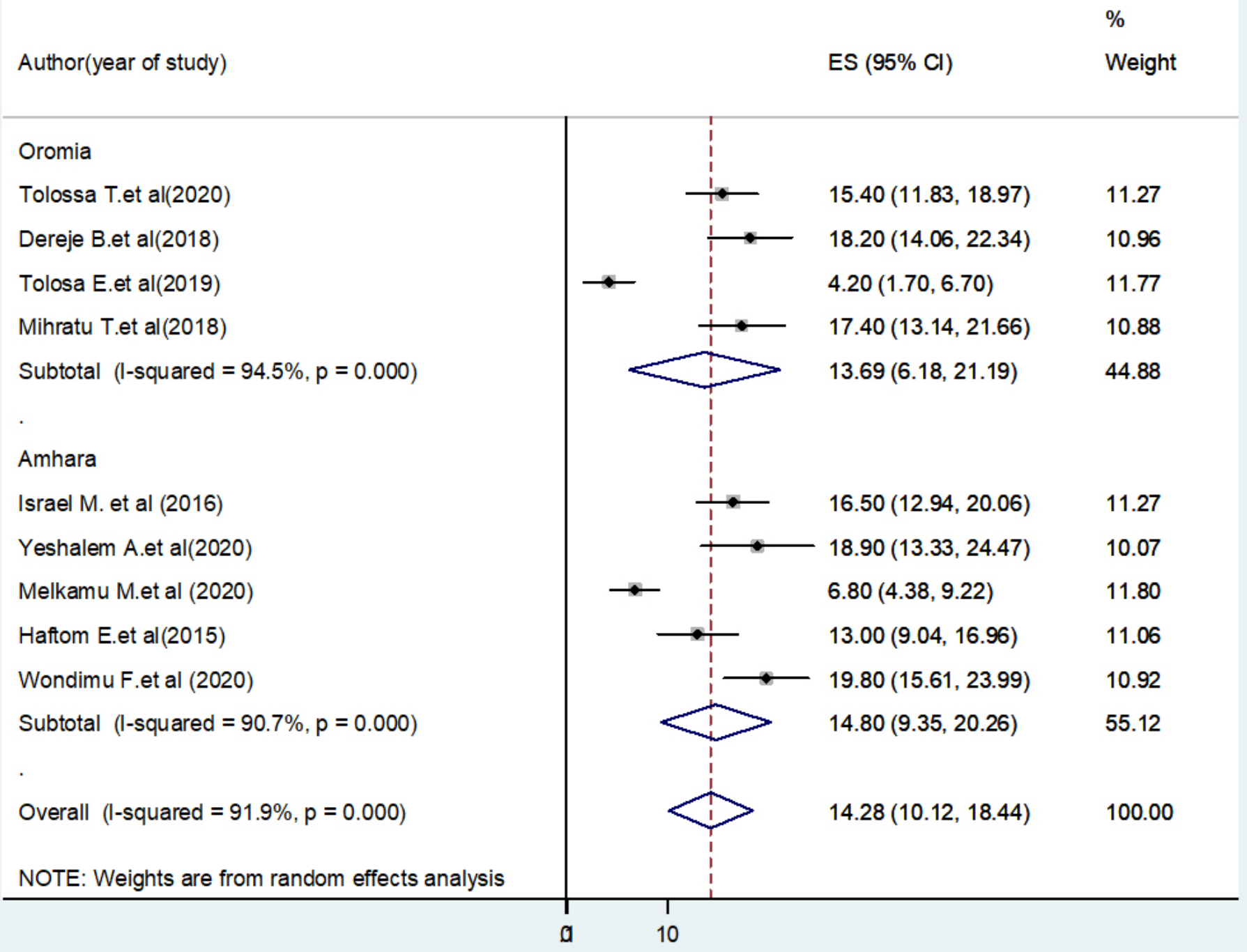

\section{Figure 5}

Subgroup analysis of the pooled prevalence of lost to follow-up among pregnant and lactating mothers living with HIV and enrolled in HIV care clinics in Ethiopia based on the study region 


\begin{tabular}{|c|c|c|c|}
\hline \multicolumn{2}{|l|}{ Author(year of study) } & \multirow[t]{2}{*}{ ES $(95 \% \mathrm{Cl})$} & $\begin{array}{l}\% \\
\text { Weight }\end{array}$ \\
\hline$>334$ & & & \\
\hline Tolossa T.et al(2020) & & $15.40(11.83,18.97)$ & 11.27 \\
\hline Israel M. et al (2016) & & $16.50(12.94,20.06)$ & 11.27 \\
\hline Melkamu M.et al (2020) & $\rightarrow$ & $6.80(4.38,9.22)$ & 11.80 \\
\hline Wondimu F.et al (2020) & & $19.80(15.61,23.99)$ & 10.92 \\
\hline Subtotal (l-squared $=92.6 \%, p=0.000)$ & & $14.49(8.35,20.64)$ & 45.26 \\
\hline & & & \\
\hline$<334$ & & & \\
\hline Yeshalem A.et al(2020) & & $18.90(13.33,24.47)$ & 10.07 \\
\hline Dereje B.et al(2018) & & $18.20(14.06,22.34)$ & 10.96 \\
\hline Tolosa E.et al(2019) & $\rightarrow$ & $4.20(1.70,6.70)$ & 11.77 \\
\hline Haftom E.et al (2015) & & $13.00(9.04,16.96)$ & 11.06 \\
\hline Mihratu T.et al(2018) & & $17.40(13.14,21.66)$ & 10.88 \\
\hline Subtotal $(1-$ squared $=93.0 \%, p=0.000)$ & & $14.17(7.60,20.75)$ & 54.74 \\
\hline & & & \\
\hline Overall $(I-$ squared $=91.9 \%, p=0.000)$ & & $14.28(10.12,18.44)$ & 100.00 \\
\hline NOTE: Weights are from random effects analysis & & & \\
\hline
\end{tabular}

\section{Figure 6}

Subgroup analysis of the pooled prevalence of lost to follow-up among pregnant and lactating mothers living with HIV and enrolled in HIV care clinics in Ethiopia based on the sample size 
ID

ES $(95 \% \mathrm{CI})$

Weight

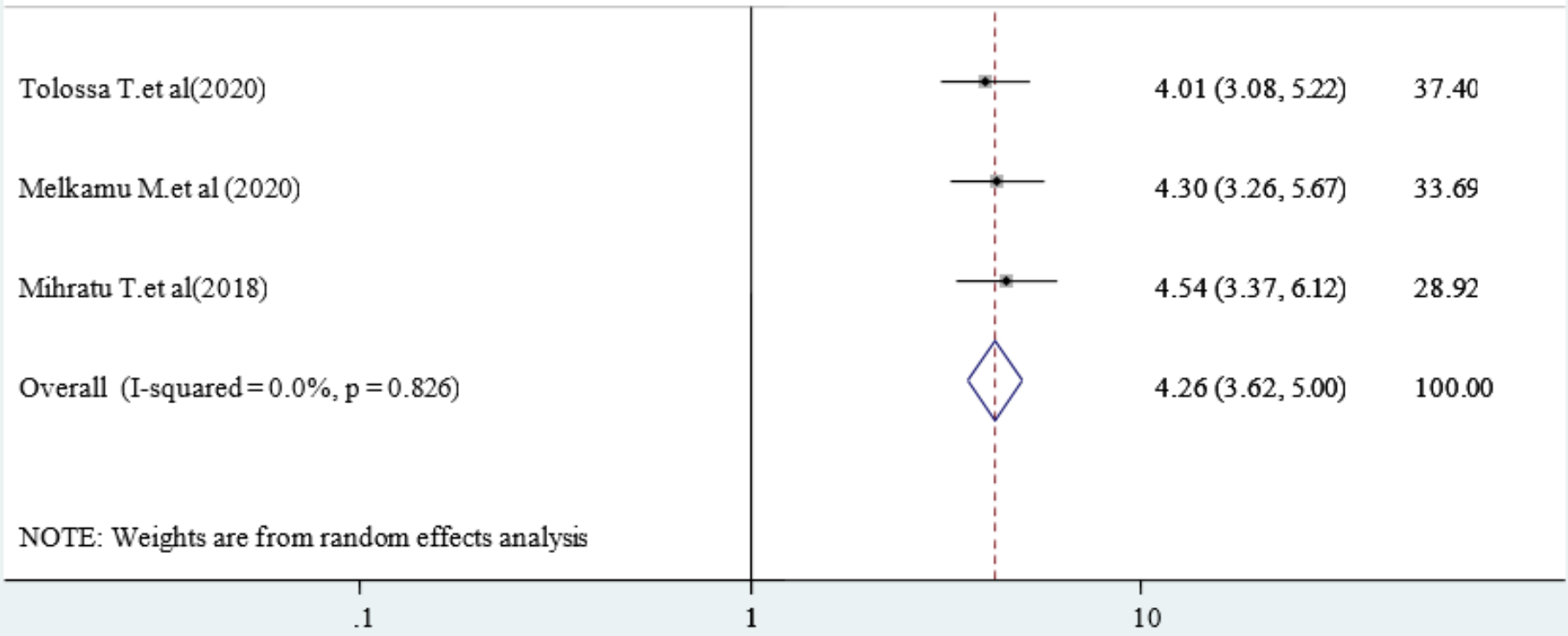

\section{Figure 7}

Relationship between women's educational level and lost to follow-up 
ID

ES (95\% CI)

Weight

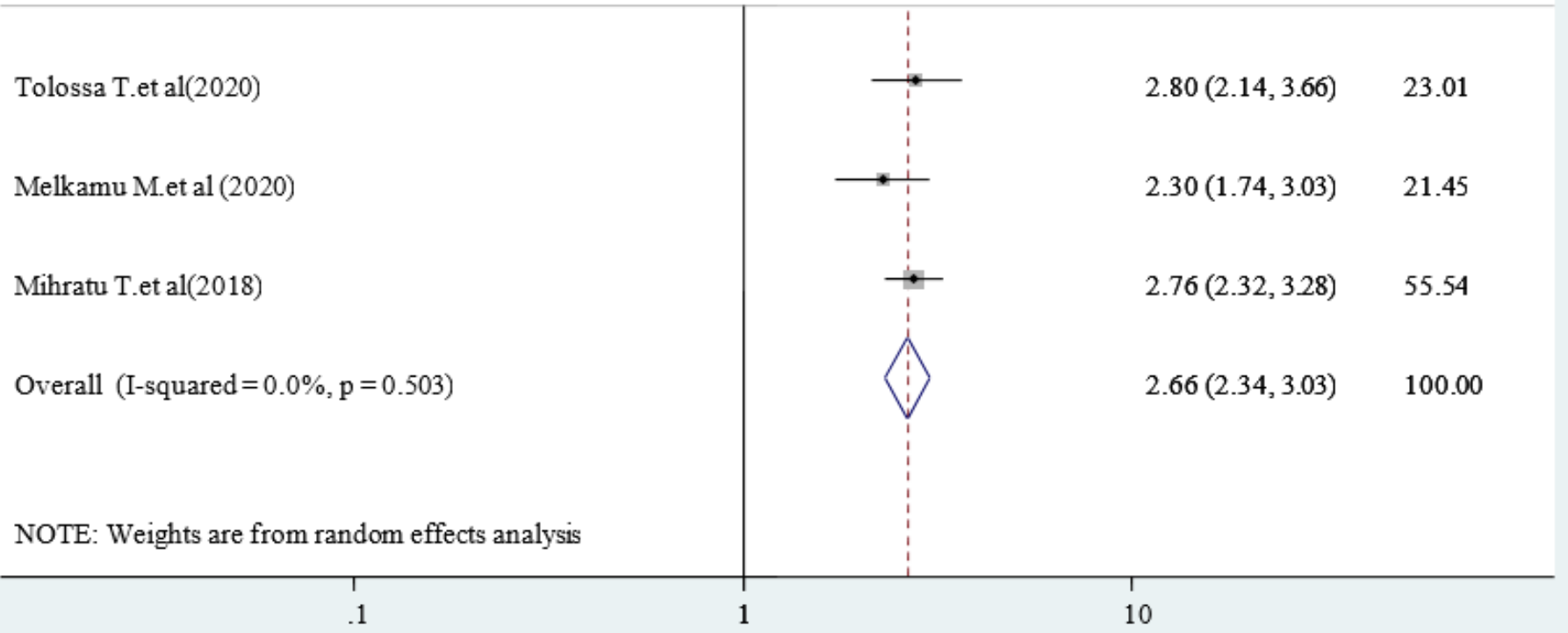

\section{Figure 8}

Relationship between women's residency and lost to follow-up 
ID

$\operatorname{ES}(95 \% \mathrm{CI})$

Weight

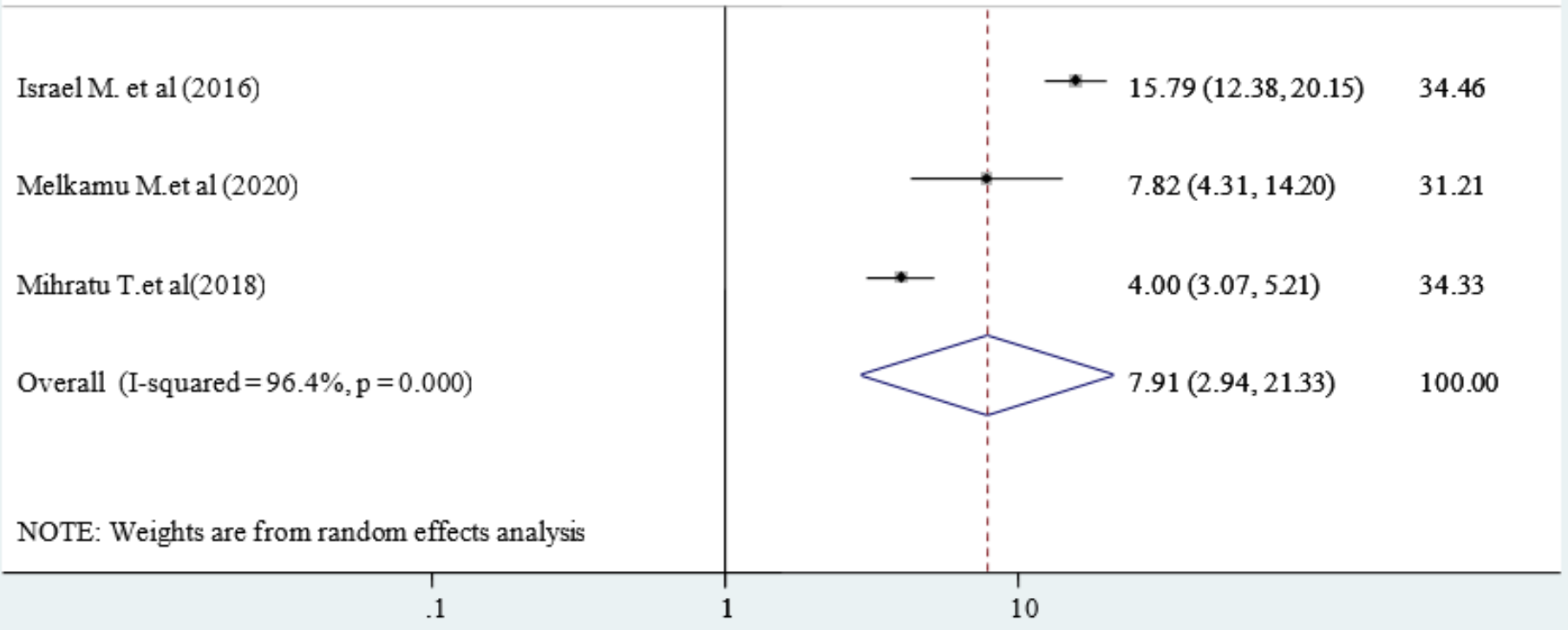

\section{Figure 9}

Relationship between experienced drug side effects and lost to follow-up 
ID

$\operatorname{ES}(95 \% \mathrm{CI})$

Weight

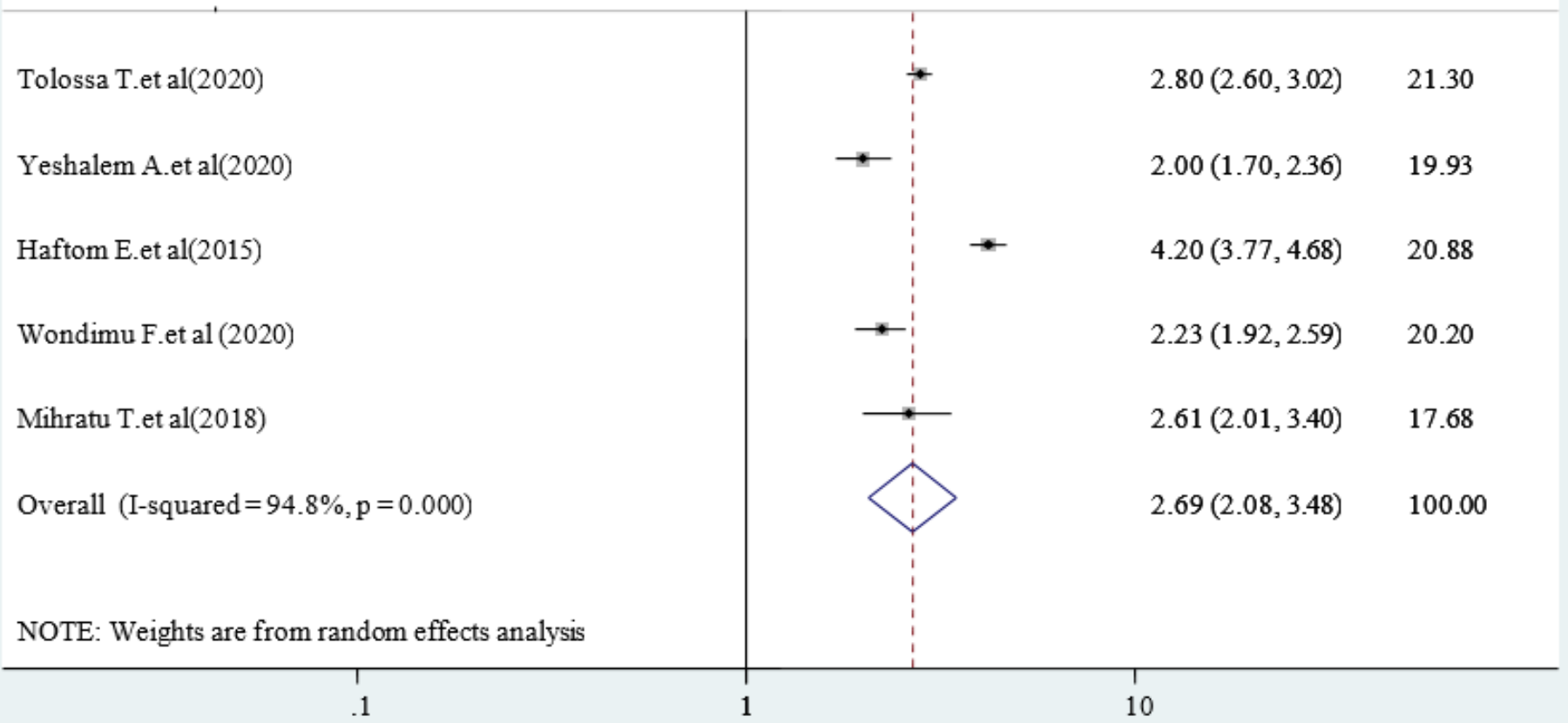

\section{Figure 10}

Relationship between disclosure of HIV status to partners and lost to follow-up 
ID

$\operatorname{ES}(95 \% \mathrm{CI})$

Weight

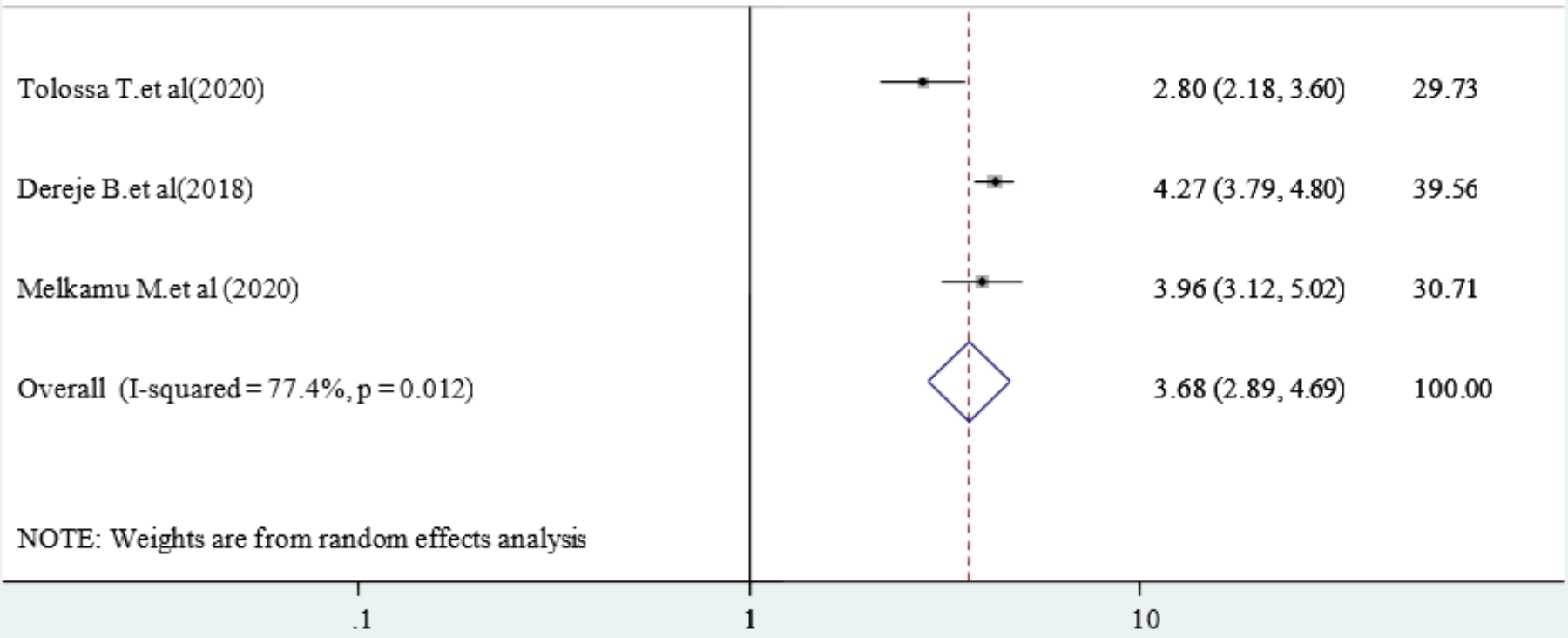

\section{Figure 11}

Relationship between poor/fair antiretroviral treatment adherences and lost to follow-up 
ID

$\operatorname{ES}(95 \% \mathrm{CI})$

Weight

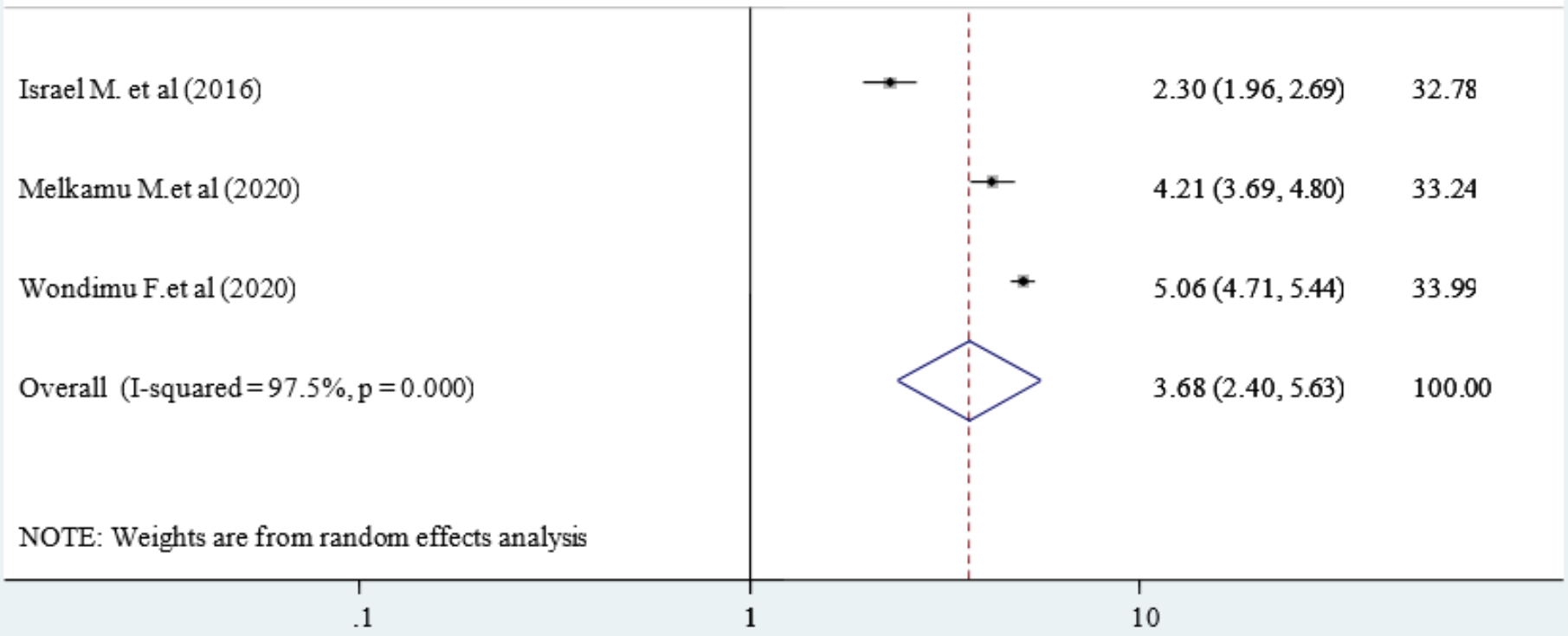

\section{Figure 12}

Relationship between knowing base line CD4 level and lost to follow-up 